\title{
Analyse der mechanischen und thermischen Beanspruchun- gen von FFZ-Reifen mit PUR-Bandage mit Hilfe der FEM- Simulation unter Variation der Gesamtradkonstruktion bei unterschiedlichen Fahrbedingungen
}

\author{
Dr. rer. nat. Gunnar Rückner, Prof. Dr.-Ing. Rainer Bruns \\ Helmut-Schmidt-Universität Hamburg \\ Lehrstuhl für Maschinenelemente und Technische Logistik
}

\begin{abstract}
Im Rahmen des Verbundprojektes InnoRad erfolgt am MTL der HSU eine computergestützte Analyse von FFZ-Rädern mit einer Bandage aus Vulkollan . Dieser Werkstoff zeigt visko-elastisches Materialverhalten. Geforscht wird an einer Abbildung der Materialeigenschaften der Kunststoffbandage aus Vulkollan $®$ und des Gesamtreifens unter den in der Praxis vorgegebenen Belastungsbedingungen für die Räder. Das thermische und mechanische Verhalten des Gesamtreifens werden mit Hilfe eines Computermodells im Rahmen einer FEM-Analyse unter Verwendung von Abaqus ${ }^{\circledR}$ untersucht. Neben der reinen Modellentwicklung wurden eine Reihe von Belastungstests mittels FEM-Simulationen durchgeführt, die das Beanspruchungsverhalten bei Aufprägung eines Momentes, der Wirkung von Querkräften, dem Drehen auf der Stelle (Lenkbewegung), sowie dem stationären Rollen beinhalten. In Kombination mit der Variation der Gesamtradgeometrie wurden auf diese Art wichtige Erkenntnisse bzgl. des Einflusses konstruktiver Variationen auf das thermische und mechanische Beanspruchungsverhalten in verschiedenen Fahrsituationen gewonnen.
\end{abstract}

\section{$1 \quad$ Einleitung}

In den letzten Jahren hat die Leistungsfähigkeit von Fluförderzeugen (FFZ) deutlich zugenommen. Höhere Geschwindigkeiten, Lasten und daraus resultierenden thermischen Belastungen führen $z u$ erhöhtem Verschleiß bei Rädern mit Kunststoffbandagen. Im Rahmen des Verbundprojektes InnoRad fand an der Professur für Maschinenelemente und Technische Logistik der Helmut Schmidt Universität Hamburg eine computergestützte Analyse von FFZ-Rädern mit einer Bandage aus Vulkollan ${ }^{\circledR}(\mathrm{Abb} .1 \mathrm{a} / \mathrm{b})$ statt. Geforscht wurde an einer Abbildung der Materialeigenschaften der PUR-Bandage und des Verbundsystems Rad, unter den in der Praxis vorgegebenen Belastungen. Diese Eigenschaften werden mit Hilfe eines Computermodells im Rahmen einer FEM-Analyse unter Verwendung der Software Abaqus ${ }^{\circledR}$ und mit Hilfe von Experimenten untersucht. 

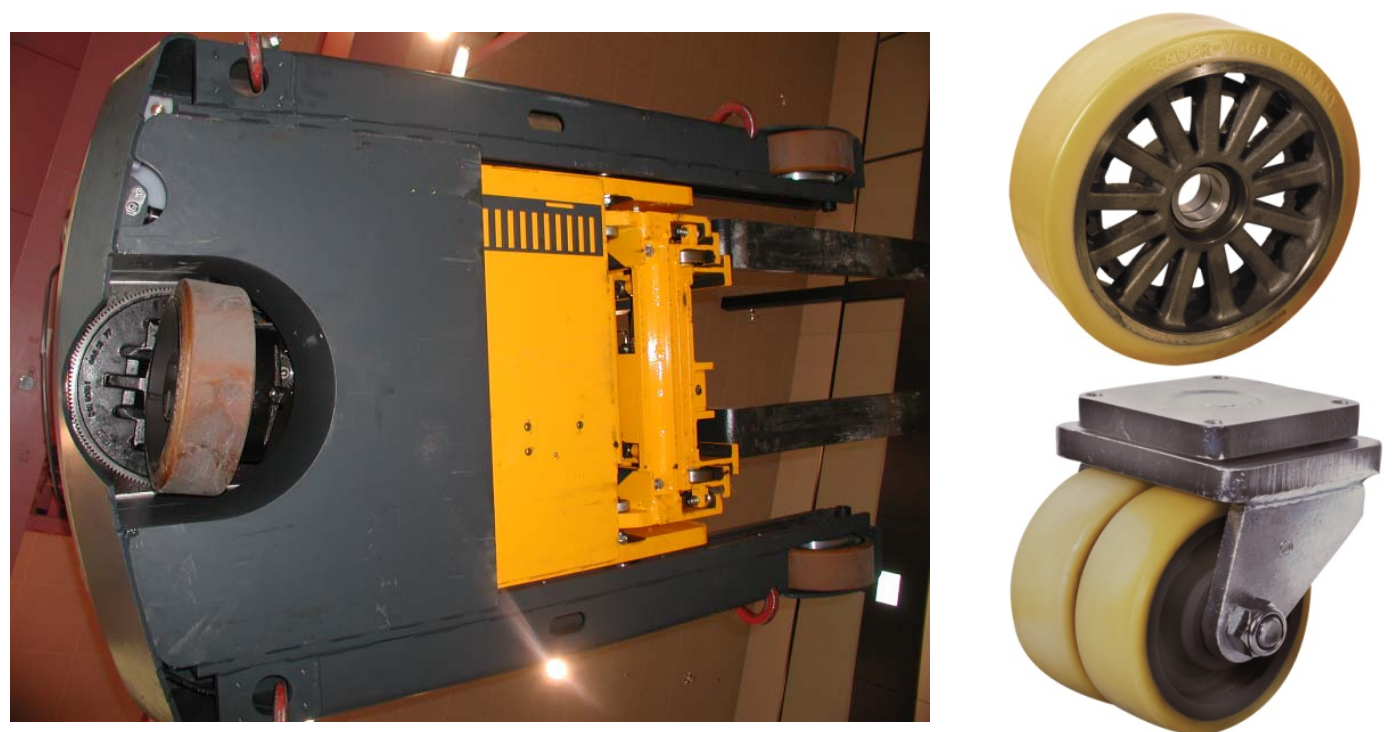

Abbildung 1a; 1b: Schubmaststapler mit Rädern mit Bandage aus Vulkollan ${ }^{\circ}$; Vulkollanräder

\section{Vulkollan ${ }^{\circledR}$ - Allgemeine Materialeigenschaften und spezielle Eigenschaften im Einsatz als Bandagenmaterial für Reifen von FFZ}

Vulkollan ${ }^{\circledR}$ zeigt visko-elastisches Materialverhalten und ist ein schlechter Wärmeleiter. Deshalb ist die im Innern erzeugte Wärme größer, als die nach Außen transportierte Wärmemenge und das Rad erwärmt sich im Rollbetrieb. Bei schnellen Fahrten oder langen Fahrstrecken kann die lokale Temperatur das Maß für die mechanische Grenzbelastung sein. Sie kann bis zum Schmelzpunkt des PURWerkstoffs ansteigen. Das Rad ist dann partiell thermisch Zerstört (Abb.2).

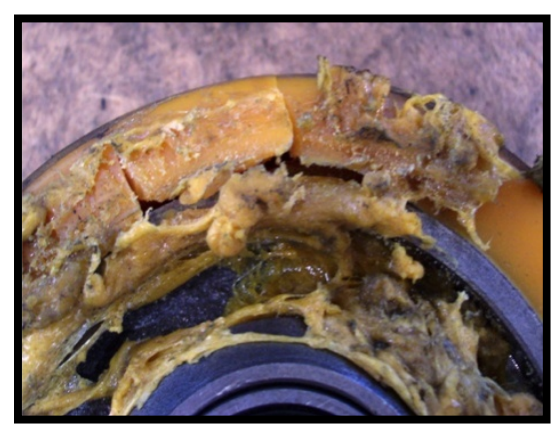

Abbildung 2: Heat Built up

Die Abhängigkeit der Materialeigenschaften der Bandage von der Temperatur führt dazu, dass sich die Werkstoffeigenschaften im Rollbetrieb ändern. Wegen des Reibkontaktes von der Fahrbahn zum Rad, treten Abhängigkeiten vom Kontaktpartner (Wärmeübergang, Reibungseffekte) auf, die in den numerischen Simulationen Berücksichtigung finden müssen. Darüber hinaus ist Vulkollan® inkompressibel. Bei stationärer Fahrt ohne Lenk- und Bremsmanöver und Stufenfahrten, ist die größte wirkende Belastung die Massenbelastung senkrecht zur Aufstandsfläche. Daraus resultiert die betraglich größte wirkende (Hauptnormal-) Spannungsbelastung (HNS), welche sich im Fahrbetrieb ebenfalls in Richtung der 
Aufstandsfläche befindet (Abb.3). Diese Ausrichtung der HNS hat sich im Rahmen der gerechneten Belastungen ( $5 \mathrm{kN}-50 \mathrm{kN})$ als unabhängig von diesen erwiesen.

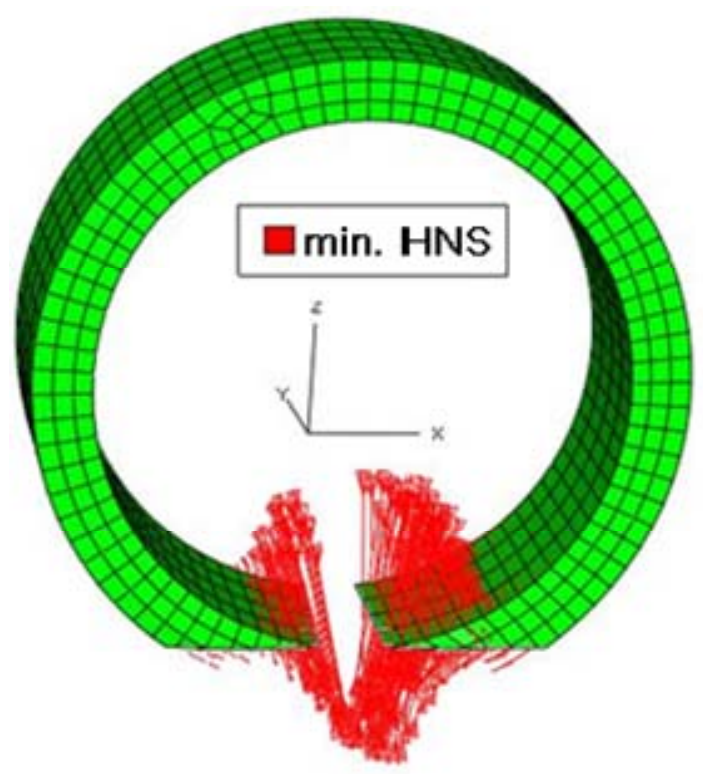

Abbildung 3: Richtung der kleinsten HNS bei stationärer Fahrt

\section{Experimente am MTL}

Neben Versuchen bei den Projektpartnern wurden am MTL eine Reihe von Experimenten zur Materialcharakterisierung vorgenommen, mit dem Ziel Materialkenndaten im betriebsrelevanten Bereich zu bekommen (uniaxiale Druckversuche von einigen $\mathrm{kN}$, temperatur- und frequenzabhängig). Präsentiert wird hier eine kleine Auswahl der durchgeführten Experimente.

\subsection{Dynamische Druckversuche zur Bestimmung von Frequenzeigen- schaften und Temperaturverhalten von Vulkollan ${ }^{\circledR}$}

Um die Erwärmung des Werkstoffs durch innere Reibung zu studieren, wurden Hydropulserversuche unter Druckbelastung (5-20kN) mit Zylinderproben (Geometrien: $40 \mathrm{~mm} \times 20 \mathrm{~mm}, 40 \mathrm{~mm} \times 40 \mathrm{~mm}, 60 \mathrm{~mm} \times 20 \mathrm{~mm}$ und $60 \mathrm{~mm} \times 40 \mathrm{~mm}$ aus Vulkollan $\circledast$ ) bei den Frequenzen $0,5 \mathrm{~Hz}, 3 \mathrm{~Hz}$ und $5 \mathrm{~Hz}$ durchgeführt. Bei typischen Fahrgeschwindigkeiten liegen die Belastungsfrequenzen eines Punktes auf der Oberfläche eines Rades im einstelligen Hertz-Bereich. Der betriebsrelevante Bereich wurde somit mit den Versuchen abgedeckt. In die Proben wurde ein Loch gebohrt, um die Innentemperatur der Probe mit einem Thermosensor messen zu können Abb. 4).

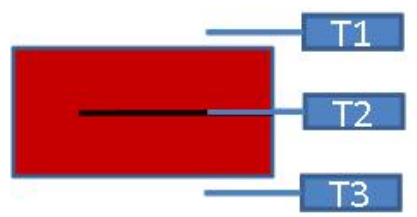

Abbildung 4: Probe mit eingeführtem Thermosensor 
Um die bei Stahlplatten verstärkt auftretende Wärmeabfuhr (Abb. 6) zu unterdrücken, wurden die Versuche zusätzlich unter Verwendung von Polyamid-Platten (PA) (Abb. 5) durchgeführt. Die Polyamid-Platten entsprechen eher den Begebenheiten der Reifen im Fahreinsatz, da die Wärmeabfuhr zwischen Probe und Polyamid realistischer ist. Die Messungen erstreckten sich jeweils über so viele Belastungszyklen, bis sich eine konstante Temperatur im Innern eingestellt hatte.
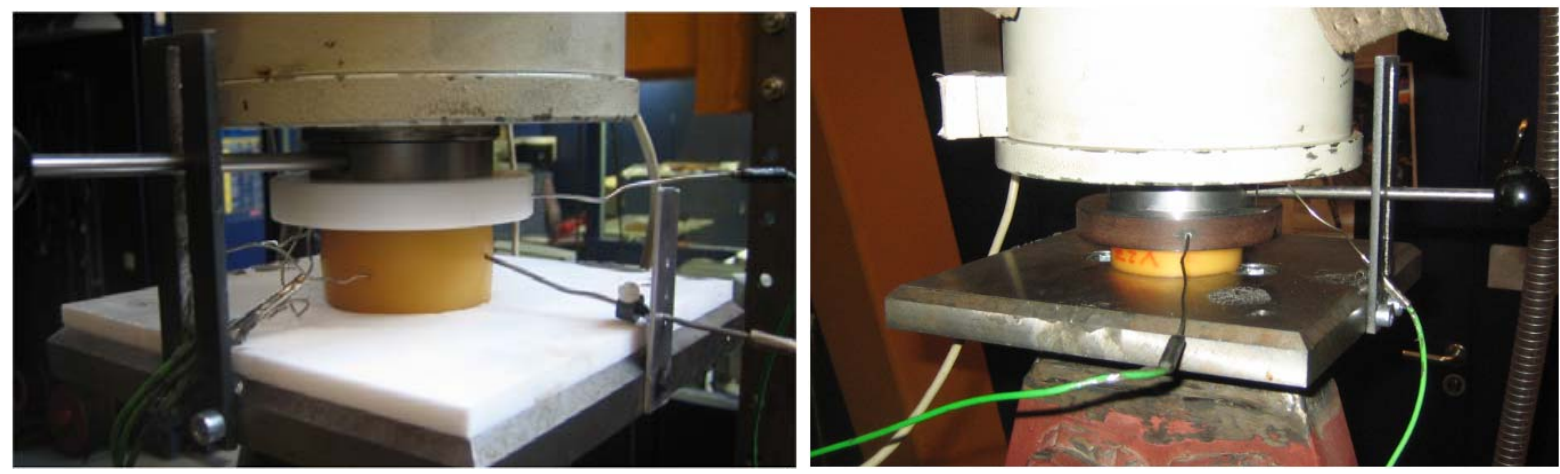

Abbildung 5: Links: Probe zwischen Polyamid; rechts: Probe zwischen Stahlplatten

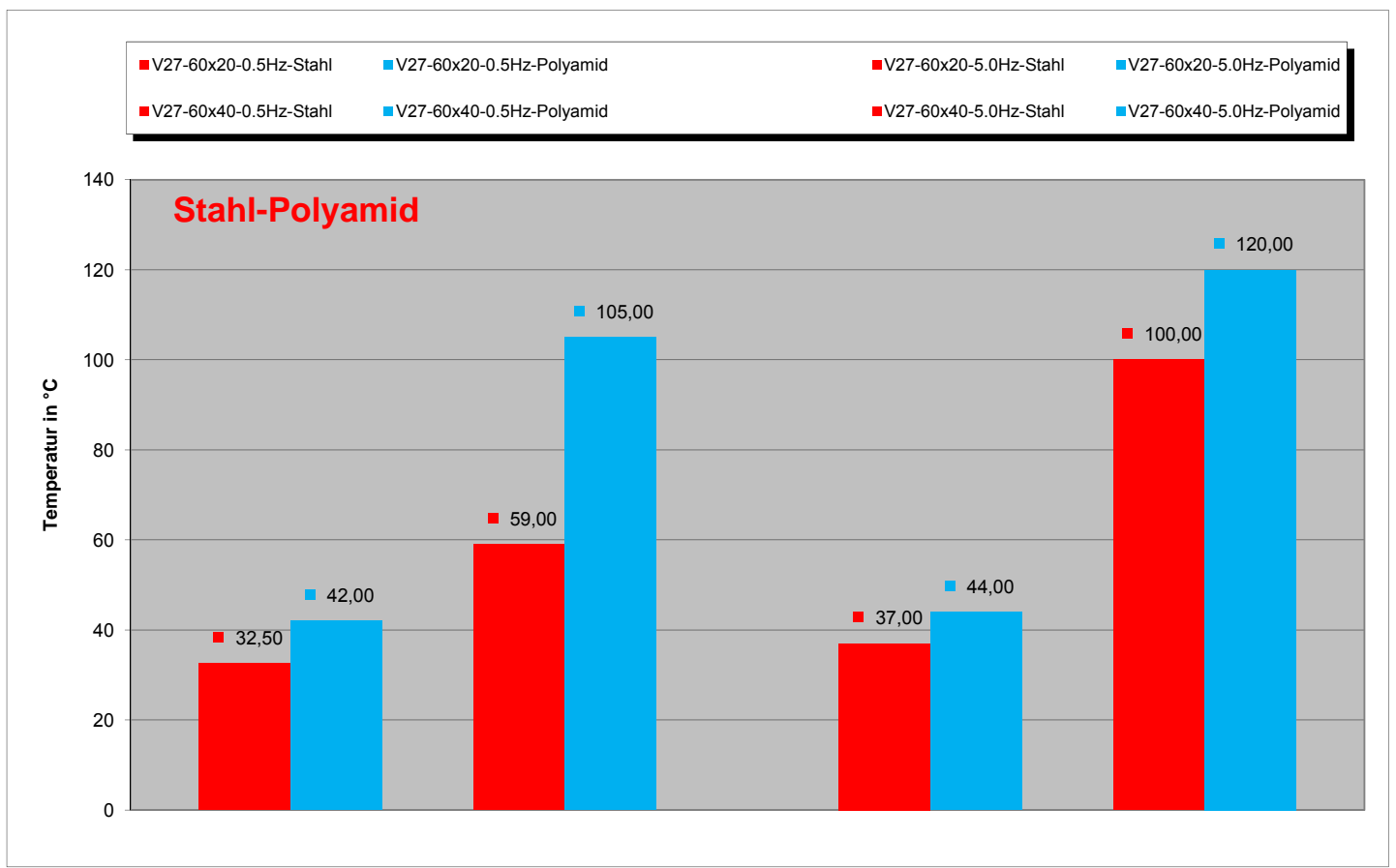

Abbildung 6: Vergleich der Peaktemperaturen zwischen Stahl und Polyamid

\subsection{Quasistatische Druckversuche}

Zur Beschreibung von nichtlinearen elastischen Effekten wurden quasistatische, uniaxiale Druckversuche durchgeführt, um mittels hyperelastischer Materialmodelle dieses Verhalten zu beschreiben. Da die Dehnungen unterhalb von $20 \%$ liegen, wurde es als ausreichend empfunden, das Verhalten mittels des Neo-Hookeschen Materialansatzes zu beschreiben. Es wurden deshalb uniaxiale Druckversuche bei RT $\left(24^{\circ} \mathrm{C}\right)$ und $100^{\circ} \mathrm{C}$ zur Bestimmung der Neo-Hookeschen Parameter durchgeführt. Für die Druckversuche wurden unterschiedliche kubische Geometrien 
genutzt. Wie in den Hydropulserversuchen gezeigt, können bei betriebsrelevanten Belastungen Temperaturen von $100^{\circ} \mathrm{C}$ und darüber entstehen. Deshalb wurde die zweite Messreihe zur Bestimmung der Materialparameter bei $100^{\circ} \mathrm{C}$ durchgeführt. Zur Bestimmung wurden alle drei Seiten der Kuben nacheinander mit 5kN 15 Zyklen lang belastet. Zwischen den einzelnen Zyklen wurden Ruhephasen von 20s eingehalten, damit das Material die Zeit hat, sich wieder in den Ursprungszustand zurückzuformen. Diesen Vorgang haben wir 3-mal durchgeführt und aus den Messwerten aus Belastung und Weg wurden mit Berücksichtigung auf die tatsächliche Probengeometrie die Spannung und die Dehnung ermittelt. Aus den Spannungs- und Dehnungswerten wurden dann unter Berücksichtigung des inkompressiblen Verhaltens von Vulkollan® die Neo-Hookeschen Parameter berechnet.

Tabelle 1: Neo-Hookesche Paramameter

\begin{tabular}{|l|c|c|}
\hline $\mathrm{C}$ in [N/mm $\left.{ }^{2}\right]$ & $24^{\circ} \mathrm{C}$ & $100^{\circ} \mathrm{C}$ \\
\hline Belastung: $5 \mathrm{kN}$ & 10.51 & 8.61 \\
\hline
\end{tabular}

\subsection{Fazit der Versuche}

Es wurde durch die Versuche nachgewiesen, dass durch die Erhöhung der Belastungsfrequenz des Materials bei gleicher Belastung sich die Temperatur erhöht. Dabei wurden Temperaturen im Bereich von $32.5^{\circ} \mathrm{C}$ bei Geometrie $60 \times 20$ und $0.5 \mathrm{~Hz}$ bis $100^{\circ} \mathrm{C}$ bei Geometrie $60 \times 40$ und $5 \mathrm{~Hz}$ gemessen. Ein Einfluss der Belastungsfrequenz auf die Stauchung der proben konnte im Rahmen der Messungenauigkeit von 10\% nicht festegestellt werden (ohne Abb.). Dies bedeutet, dass sich die Temperatur in der Bandage der Reifen bei schnellerer Fahrt dramatisch erhöhen wird.

\section{$4 \quad$ Gesamtmodellbildung}

Realisiert wurde ein Thermomechanisches Modell (siehe Ablaufskizze - Abb. 7). Dabei wurde für die mechanische Beschreibung eine Kopplung des allgemeinen Maxwellmodells für die Beschreibung des visko-elastischen Verhaltens mit hyperelastischen Modellen vorgenommen. Das nichtlineare elastische Verhalten wird dabei durch hyperelastische Materialmodelle beschrieben. Dabei wird eine Formänderungsenergiefunktion aufgestellt, die für homogene, isotrope Materialien im Hauptdehnungszustand die allgemeine Formt besitzt.

$$
\mathrm{U}=\mathrm{U}(\mathrm{I}, \mathrm{Il}, \mathrm{III}) \text { oder } \mathrm{U}=\mathrm{U}\left(\lambda_{1}, \lambda_{2}, \lambda_{3}\right),
$$

hier sind I,II,III die Hauptinvarianten des Rechten Cauchy-Green-Tensors und $\lambda_{\mathrm{i}}$ die Dehnungen Im einfachsten Fall können dessen Materialparameter mittels uniaxialer Versuche bestimmt werden können. Ein Beispiel hierfür ist das kompressible NeoHookesche Materialgesetz, dessen Materialparameter im Abschnitt Experimente bestimmt wurden. Das Neo-Hookesche Modell kann bevorzugt bei kleineren 
Dehnungen im Bereich von weniger als etwa 30\% Dehnung angewendet werden. Dieses Materialverhalten liegt in unserem Falle vor.

$$
U=C(I-3)+1 / D_{1}(J-1)^{2}
$$

mit der Determinante $\mathrm{J}$ des Verzerrungstensors, I erste Hauptinvariante des linken Cauchy-Green-Tensors, $C$ Anfangs Schermodul und $2 / D_{1}=K_{0}$ Anfangsbulkmodul. Im Fall von inkompressiblen Materialien - wie dem von uns untersuchten Vulkollan - kann der volumetrische Anteil vernachlässigt werden. Das implementierte Modell beinhaltet die sequentielle Berechnung der mechanischen Eigenschaften und die daraus resultierenden Wärmequellen, die dann in einem Wärmeleitungsabschnitt zur Bestimmung der Temperaturen im Innern des Reifens verwendet werden (Prinzipskizze - Abb. 7). Zu diesem Zweck werden die Vernetzung und Aufbringung der Lasten komplett mittels eines entwickelten Inputfilegenerators automatisch erzeugt. Der entwickelte Inputfilegenerator bildet auch die Basis für die Inputfilegeneratoren, mit denen parametrische Variationen der Radgeometrie (Felgen - und Bandagengeometrie) möglich sind (Abb. 8).

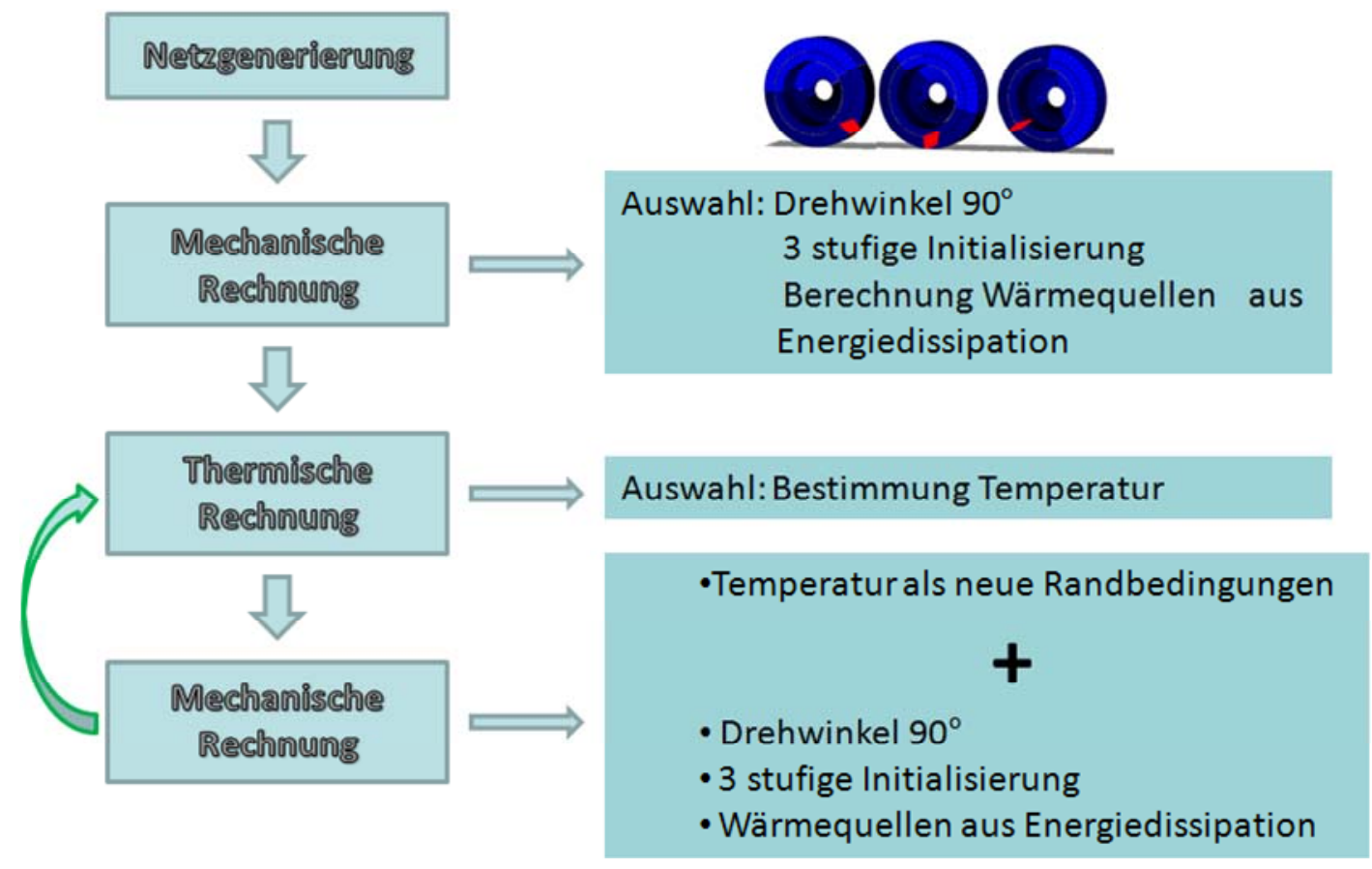

Abbildung 7: Ablaufskizze der Rechnungen

Die Wärmequellen werden in der mechanischen Teilrechnung innerhalb eines vorher festgelegten Bereiches bestimmt (roter Bereich in den Rädern [hier Scheibe, Abb. 7]). Die Lastdaten in diesem Bereich werden zeitlich integriert und dann in der zweiten Teilrechnung - einer Wärmeleitungsrechnung - zur Temperaturfindung verwendet. Da die Vernetzung komplett selber vorgenommen werden, sind alle Knoten-, respektive Elementnummerierungen bekannt. 
Neben der Entwicklung des Materialmodells wurden parametrisierte Inputfilegeneratoren entwickelt, die es per Knopfdruck erlauben, eine Vielzahl unterschiedlicher Radkonstruktionen auf deren Tauglichkeit hin zu untersuchen (Abb. 8). Ausgehend vom Standardrad (S-Rad), wurden IG mit variierter Felgenposition (Kraftfluss! V1)), Varianten mit einer L-Klammerung (V2) und zwei Varianten mit schrägem Bandagen-Felgen-Übergangsprofil (V3 und V4) entwickelt. Für die mit den IGs erstellbaren Radkonstruktionen wurde ein breiter Katalog an Lastfällen durchgerechnet (Variation der Achslast, Querkrafteinfluss, Einfluss der Fahrgeschwindigkeit, ...).
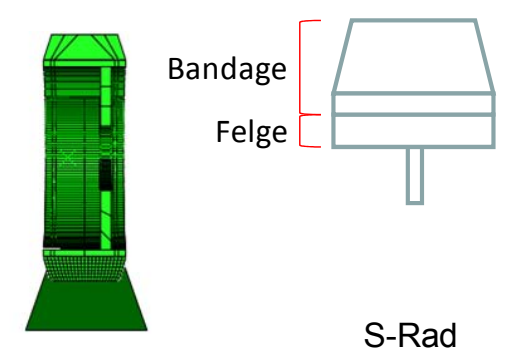

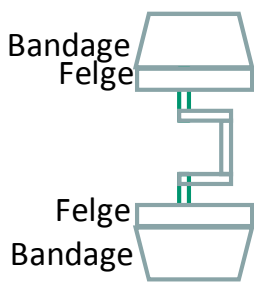

Variante 1 (V1)

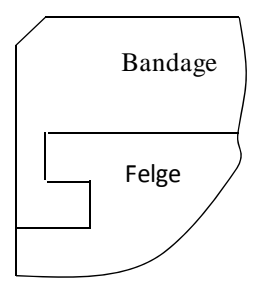

Variante 2 (V2)

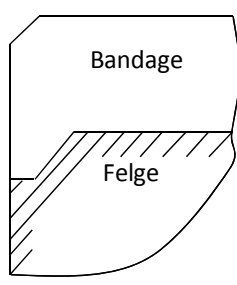

Variante 3 (V3)

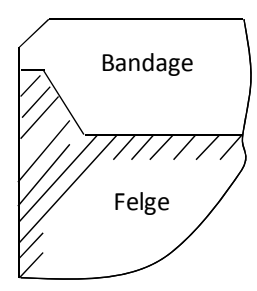

Variante 4 (V4)

Abbildung 8: Prinzipskizzen erstellbarer Radkonstruktionen

\section{$4 \quad$ Numerische Fallstudien - Auswahl}

Im Rahmen des Projektes wurde eine große Vielfalt von geometrischen, Kontaktund Belastungsparametern in den numerischen Simulationen in Abaqus ${ }^{\circledR}$ variiert, um möglichst viele Einflüsse auf die Radbelastungen aufdecken zu können. Dazu zählt eine Variation des Reibbeiwertes. Dargestellt in werden nur Ergebnisse mit gerechneten Reibbeiwerten von $\mu_{r}=0.36$ und $\mu_{r}=0.6$. Gerechnet wurden Geschwindigkeitsvariationen im Bereich von 1-20km/h unter Variation der Achslast von $9 \mathrm{kN}-49 \mathrm{kN}$. Dargestellt werden exemplarisch nur Rechnungen mit $34 \mathrm{kN}$ bei einer Fahrgeschwindigkeit von $16 \mathrm{~km} / \mathrm{h}$. Auch bei den Rechnungen zu den Belastungskollektiv wurde eine Auswahl getroffen und es wird exemplarisch nur Vergleichsrechnungen zwischen V2 und S-Rad-Variante und eine Studie zum Einfluss von Radbreite, Bandagendicke (BD) und Felgendicke (FD) gezeigt. Alle Diagramme enthalten eine ausführliche Beschriftung, die die jeweiligen relevanten Parameter enthalten.

\subsection{Einfluss der Radbreite}

Die Verringerung der Radbreite hat erwartungsgemäß in allen Fällen zu einer Erhöhung der Beanspruchung geführt. Diese Aussage gilt sowohl bzgl. der jeweils korrespondieren Räder, d.h. bei gleicher Bandagendicke und gleicher Felgendicke (Abb. 9 - 10) aber unterschiedlicher Radbreite, als auch absolut gesehen über alle geometrischen Variationen. Bei gleichen von außen aufgeprägten Lasten, wurde bei den geringeren Radbreiten eine Symmetrisierung der Beanspruchungen erwirkt (ohne Abb.). Da das allgemeine Spannungsniveau allerdings deutlich höher als bei 
den breiteren Radvarianten ausfällt, ist dies nicht weiter von Belang. Die Balkendiagramme zeigen die Peakvalues der Zug- und Druckspannungen. Tendenziell zeigen die Schubspannungen ein ähnliches Verlaufsverhalten wie die Druckspannungen, weshalb auf eine Darstellung bzgl. der Peakvalues verzichtet wurde. Der Einfluss der Radverbreiterung scheint degressiv zu verlaufen, was man an den Veränderungen der Spannungsniveaus im Vergleich der Radbreite $114 \mathrm{~mm}$ zu 136mm sehen kann. Man sollte also darauf achten, eine kritische Radbreite nicht zu unterschreiten. Die kritische Radbreite ist allerdings von der Höhe der Belastung abhängig (ohne Abb.) - hiermit ist sowohl die Traglast gemeint - als auch die Belastungsart (z.B. Kurvenfahrt, stationäres Rollen, ...).

\subsection{Einfluss der Bandagendicke}

Schub und Druckspannungen durchlaufen tendenziell ein schwach ausgeprägtes Minimum nahe der BD des S-Rades von 35mm. Zugspannungen verhalten sich dem entgegengesetzt, d.h. sinken eher mit geringerer Kissenstärke der Bandage (Abb. 9 10). Der hochbelastete Bereich ist bei dickeren Bandagen größer. Wie man den Abb. 11 entnehmen kann, ist auch die thermische Belastung bei betriebsrelevanten langen Belastungsdauern bzgl. der Bandagendicke dem Verhalten der Druck- und Schubspannungen entgegengesetzt. Es liegt bzgl. der durchgeführten Geometrischen und Lastvariationen also ein Zielkonflikt bzgl. der optimalen Bandagendicke bzgl. der Optimierung von Druck- und Schubspannungen auf der einen Seite und Zugspannungen und thermischer Belastung auf der anderen Seite vor. Vulkollan $®$ besitzt eine sehr schlechte Wärmeleitfähigkeit. Bezogen auf alle Spannungsbeanspruchungen gilt, dass je dünner die Bandage ist, desto höher ist die Gesamtspannungsbeanspruchung (bei stationärer Fahrt) und damit auch die Energiedissipation, die in einer Temperaturerhöhung resultiert. Wegen der schlechten Wärmeleiteigenschaft von Vulkollan $®$, respektive der im Vergleich dazu guten Wärmeleiteigenschaft des Felgenmaterials, wird, sobald sich die Erwärmungszone bis zur Felge hin ausgebreitet hat, die Wärme bei dünneren Bandagen schon bei kürzeren Betriebszeiten besser abtransportiert. Dies führt bei längeren Betriebszeiten zu einer geringeren Temperaturbeanspruchung bei den dünneren Bandagen im Vergleich zu den dickeren Bandagen.

\subsection{Einfluss der Felgendicke}

Die Veränderung der höchsten Beanspruchungswerte (Balkendiagramme (Abb. 9 10) für alle Spannungsbeanspruchungen variiert nur schwach. Eine Ausnahme bildet hierbei die Spannungswerte bei besonders dünnen Bandagen (Kombinationseffekt!). Dickere Felgen sorgen für eine Symmetrisierung der Beanspruchungsverteilungen. Der Einfluss der Felgendicke auf die Verbesserung bzgl. der Beanspruchung scheint degressiv zu verlaufen, d.h. dass Ausmaß der Verbesserung nimmt mit steigender Felgendicke ab. Da die Veränderung sich auf geringem Niveau zu bewegt, wird sie nicht weiter betrachtet. 
Die in den Balkendiagrammen abgebildeten BD sind jeweils die kleinste $(10 \mathrm{~mm})$, die Standardgröße $(35 \mathrm{~mm})$ und die größte $(50 \mathrm{~mm})$ gerechnete $B D$ - für die drei gerechneten Breiten $(86 \mathrm{~mm}, 114 \mathrm{~mm}$, und $136 \mathrm{~mm})$. Dargestellt sind jeweils die Werte für die kleinste FD $(10 \mathrm{~mm})$ und größte FD $(60 \mathrm{~mm})$. Die Werte der mittleren BD und FD bewegen sich jeweils innerhalb von den dargestellten Werten. Den Einfluss der Radbreite, kann man durch Vergleich jeweils korrespondierender Reifengeometrien (gleiche BD und FD) bei Variation der Breite ablesen. Den Einfluss der BD-Variation erkennt man durch Vergleich der Werte innerhalb einer Radbreitenblockes, jeweils zu demselben FD-Wert. Die BD nehmen - innerhalb eines Radbreiteblocks - jeweils von links nach rechts ab. Den FD-Einfluss kann man letztendlich jeweils durch Vergleich zweier direkt benachbarter Werte, also bei identischer BD zu jeder Radbreite entnehmen.

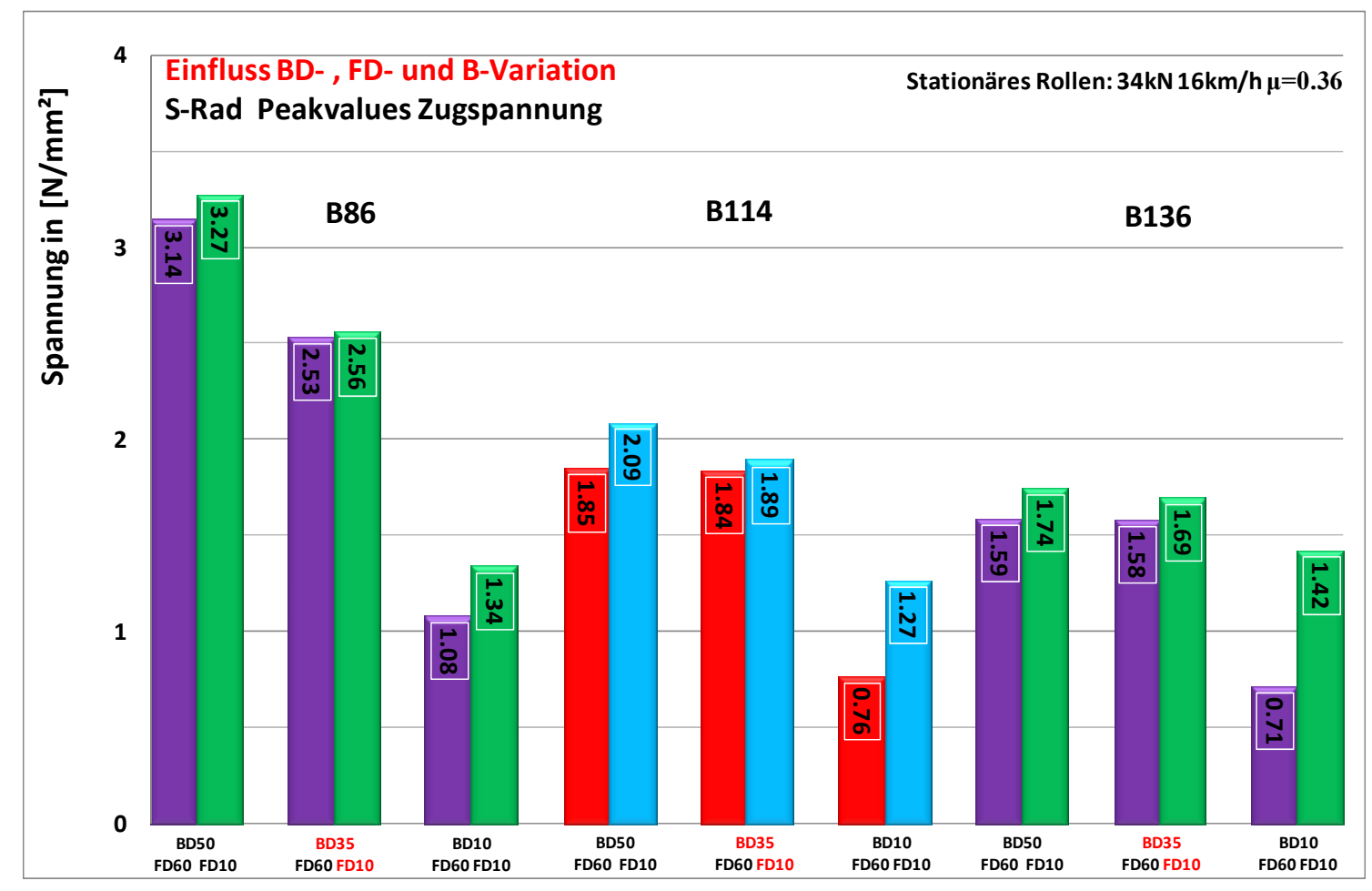

Abbildung 9: Peakvalues Radbreiten-, BD- und FD-Variation - Zugspannungen 


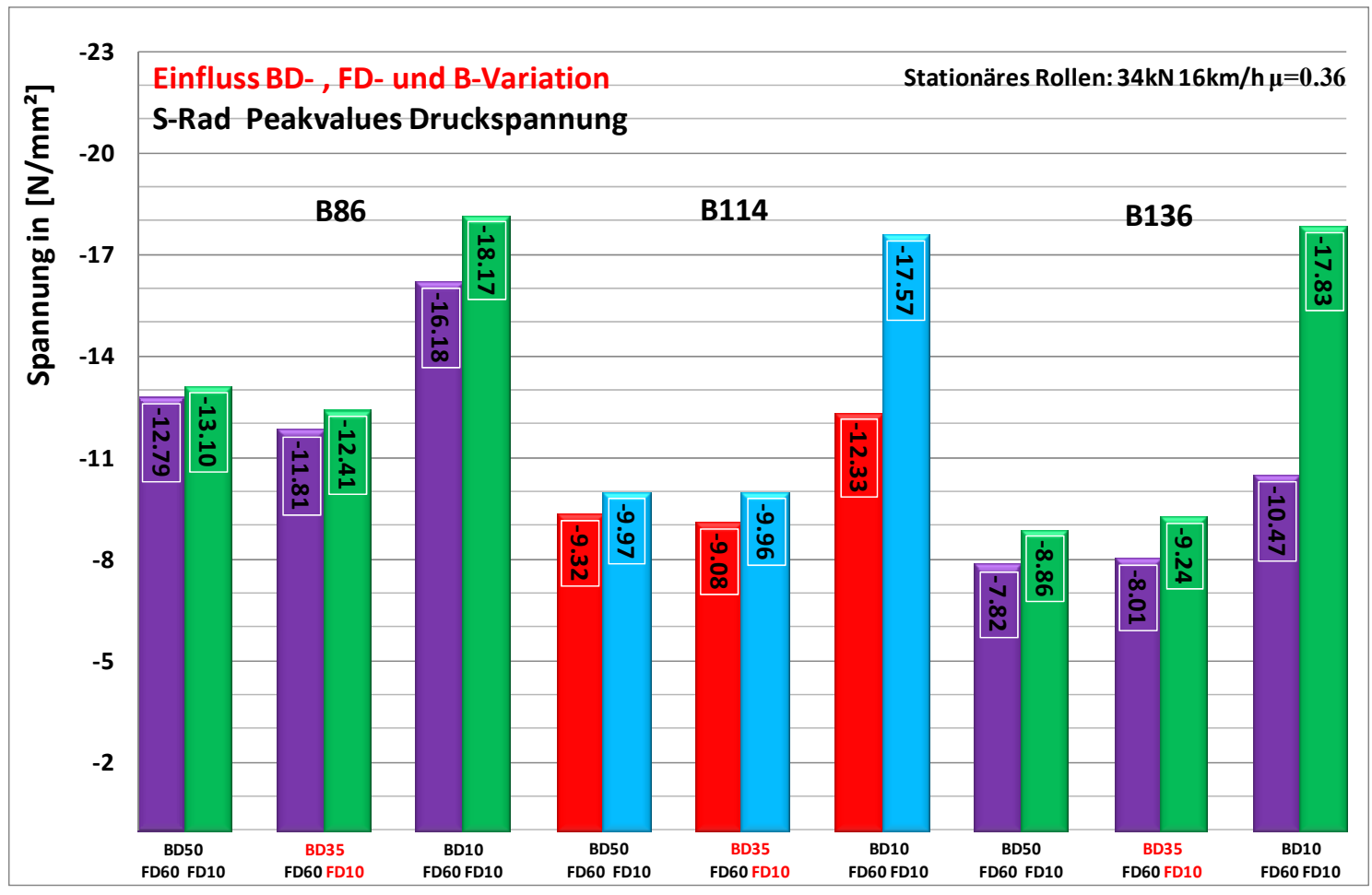

Abbildung 10: Peakvalues Radbreiten-, BD- und FD-Variation - Druckspannungen
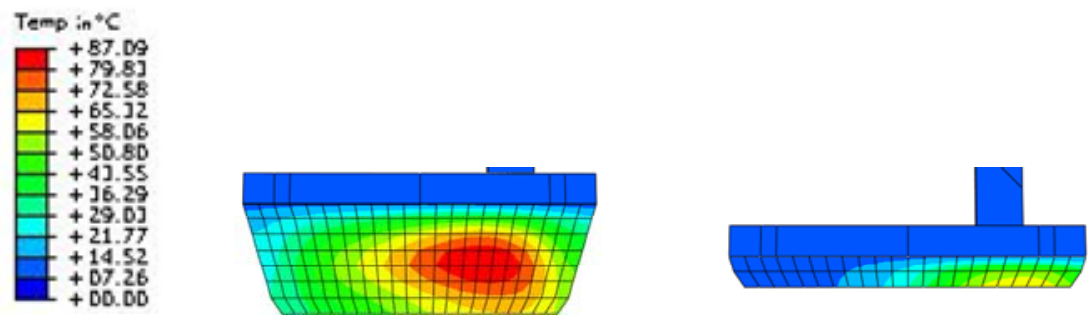

Lange (betriebsrelevante) Zeitskala
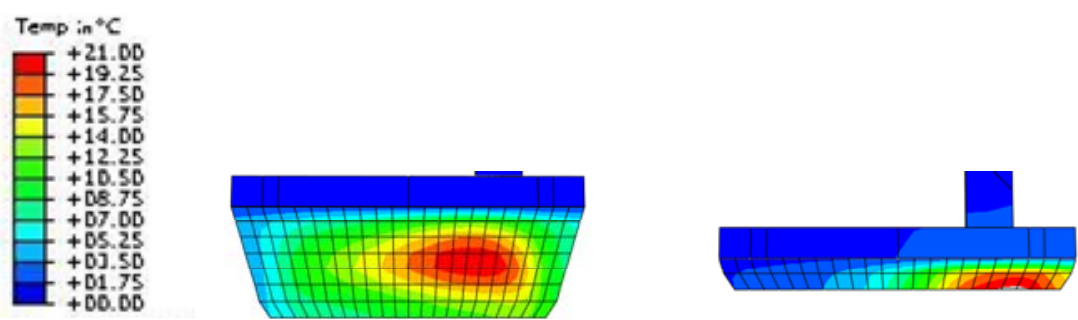

Kurze Zeitskala

Abbildung 11: Temperaturverteilung bei kurzer und langer Wärmeproduktion 


\subsection{Exemplarischer Vergleich zwischen V2 und S-Rad}

Nachfolgend wird ein kurzer Vergleich zwischen der Klammerungsgeometrie und einem Standardrad gezeigt. Exemplarisch wurde die Schubspannung herausgegriffen. Die Settingdaten sind:

Vertikale Belastung $-25 \mathrm{kN}$, Fahrgeschwindigkeit $-10 \mathrm{~km} / \mathrm{h}$,

Querkräfte $-F_{Q}=2.9 \mathrm{kN} \& F_{Q}=7.4 \mathrm{kN}$, Reibbeiwert $-\mu_{r}=0.6 \rightarrow F_{Q-M A X}=14.7 \mathrm{kN}$

Künstliche Versteifung der Felge: Variation der Felgensteifigkeit um den Faktor 2

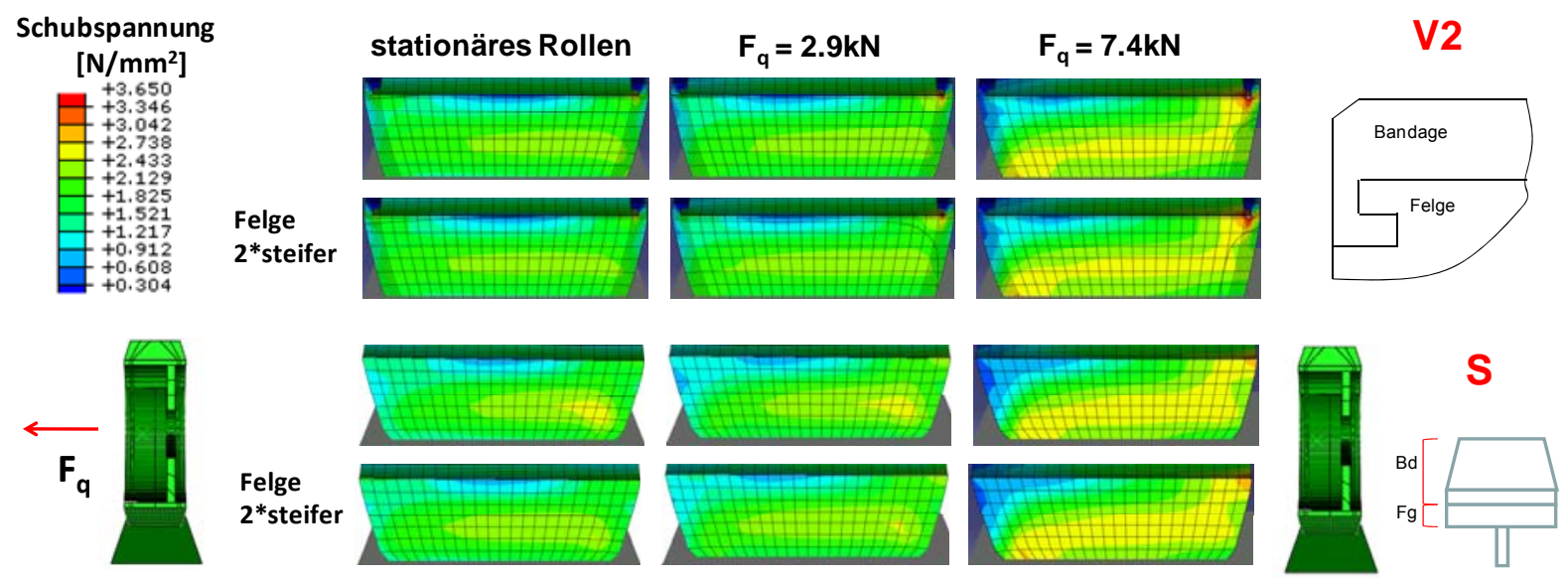

Abbildung 12: Querschnittsbilder der Radbandagen für die Geometrien V2 (oben) und S-Rad (unten). Dargestellt ist die Schubbeanspruchung. Die Spannungsskala gilt für alle Schnittbilder.

Man erkennt eine deutliche Reduzierung der Ausprägung der hochbelasteten Zonen im Innern des Bandagenmaterials durch die Klammerung (V2) gegenüber dem SRad. Dies tritt sowohl bei den gezeigten Schubspannungen als auch bei den Druckspannungen auf (ohne Abb.). Höhere Felgensteifigkeiten führen zu einer größeren Symmetrie der hochbelasteten Zonen. Hohe Querkräfte $(=$ enge Kurvenfahrten) erhöhen die Beanspruchungen signifikant. Peakvalues der Spannungen scheinen bei den V2-Rädern im Bereich der Klammerung vorzukommen, allerdings muss hier der eckigen Simulationsgeometrie Rechnung getragen werden. 


\section{$5 \quad$ Zusammenfassung und Fazit}

Präsentiert wurde ein Teil der Arbeit des MTL im Rahmen des Verbundprojektes InnoRad. Die Arbeiten am MTL beliefen sich dabei auf experimentelle Bestimmung von Materialkennwerten, über die Materialmodellentwicklung und Implementierung bis hin zum Einsatz des Gesamtradmodells zur Überprüfung konstruktiver Radvarianten. Die nachfolgenden Aussagen beziehen sich auf die hier präsentierte Untermenge an numerischen Simulationen und Experimenten.

\section{Einfluss BD (dicke Bandagen)}

- Größerer Bereich hoher Spannungen

- Negative Auswirkung auf Zugspannungen

- Thermische Beanspruchung steigt (bei schlechter Wärmeleitungseigenschaft des Bandagenmaterials)

\section{Einfluss FD (dicke Felgen)}

- Bessere Wärmeabfuhr (bei guten Wärmeleitungseigenschaften der Felge und (!) guter Luftzufuhr, d.h. eine geringe Radkapselung ist dafür notwendig).

- Positiver Einfluss auf Schubspannungen - Verbesserung stark degressiv mit FD.

- Symmetrisierung der hochbelasteten Zone (Vorsicht, damit wird die Wärmeproduktion auch mittig verlagert, was bei schlechten Wärmeleitungseigenschaften zu einer Verschlechterung bzgl. der thermischen Beanspruchung führen kann.).

\section{Einfluss Radbreite}

- Tendenziell degressiv wirkender, positiver Effekt.

\section{Einfluss Querkraft}

- Richtung (Links- oder Rechtskurve) der Querkraft beeinflusst deutlich Ort und Höhe der höchsten Beanspruchungen.

- Amplitude der Querkraft beeinflusst deutlich die Lage und Ausdehnung der hochbelasteten Zonen. Je weiter man sich der Rutschgrenze nähert, desto mehr werden die hochbelasteten Zonen in den Randbereich der Räder verlagert.

Neben den hier präsentierten Ergebnissen, wurden eine Reihe weiterer Analysen vorgenommen, die für die Einschätzung günstiger Radgeometrievarianten wertvoll waren. 